14,15

\title{
Гидродинамические течения в микроразмерных жидкокристаллических ячейках с ориентационными дефектами
}

\author{
(C) A.B. Захаров \\ Институт проблем машиноведения РАН, \\ Санкт-Петербург, Россия \\ E-mail: alexandre.zakharov@yahoo.ca
}

(Поступила в Редакцию 14 марта 2018 г.

В окончательной редакции 26 марта 2018 г.)

\begin{abstract}
Численными методами, в рамках нелинейного обобщения классической теории Эриксена-Лесли, с учетом термомеханических вкладов как в тензор напряжения, так и в вязкий момент, действующий на единицу объема жидкокристаллической фазы (ЖК-фазы), исследовано влияние ориентационного дефекта (ОД) на процесс формирования вихревого потока $\mathbf{v}(t, \mathbf{r})$, возникающего в микроразмерной жидкокристаллической (ЖК) ячейке под действием сфокусированного лазерного излучения. Анализ полученных результатов показал, что в ,дефектной“ ЖК-ячейке с ОД, расположенным на нижней ограничивающей поверхности, на которую было сфокусировано лазерное излучение, вблизи ОД формируется вихревой поток, вращающийся по часовой стрелке. Скорость вращения этого потока на два порядка больше скорости вращения вихря, формирующегося при тех же условиях в „бездефектной“ ЖК-ячейке и вращающегося против часовой стрелки.
\end{abstract}

Работа выполнена при финансовой поддержке РФФИ (грант № 16-02-00041a) и Минобрнауки (грант 3.11888.2018/11.12, „Физические основы применения микрофлюидики в оптоэлектронике“).

DOI: 10.21883/FTT.2018.09.46407.068

\section{1. Введение}

Молекулярные жидкости и жидкие кристаллы (ЖК), инкапсулированные в микро- и нанолитровые объемы, находят применение в разнообразных сенсорах и датчиках, используемых в биотехнологических приложениях, медицине и фармакологии, а также в иммерсионной литографии. Один из способов, позволяющий манипулировать такими микроразмерными системами, основывается на использовании электрического поля (электрокинетика) [1]. Другой способ, позволяющий транспортировать микролитровые объемы молекулярных жидкостей, основан на формировании разности градиентов поверхностного натяжения (ГПН) на границах раздела жидкость/газ и жидкость/твердое тело [2]. Эта разность ГПН может быть создана посредством локального разогрева ограниченного объема жидкости, например, с помощью сфокусированного лазерного излучения. Эти вышеописанные методы транспортировки микроразмерных объемов одинаково применимы как для молекулярных жидкостей, так и для манипулирования микролитровыми объемами ЖК-материалов. Отличительной особенностью ЖК-систем от изотропных молекулярных жидкостей является то, что при определенных термодинамических условиях в ЖК-системах формируется упорядочение молекул, которое описывается полем директора $\hat{\mathbf{n}}$ [3]. Ранее было показано, что взаимодействие градиентов поля директора $\nabla \hat{\mathbf{n}}$ и температуры $\nabla T$ ведет к формированию устойчивого гидродинамического потока $\mathbf{v}$ ЖК-материала [4]. Величина этого потока пропорциональна сдвиговой составляющей термомеханического вклада $\sigma_{z x}^{t m}$ в тензор напряжения $(\mathrm{TH}) \quad v \sim \frac{d}{\eta} \sigma_{z x}^{t m}$, где $\sigma_{z x}^{t m} \sim \xi \frac{\triangle T}{d^{2}}, \quad \eta-$ сдвиговая вязкость ЖК-материала, $\triangle T=T_{2}-T_{1}>0$, а область температур $\left[T_{2}, T_{1}\right]$ находится в пределах стабильности нематической фазы толщиной $d$, в то время как $\xi-$ термомеханическая постоянная [5]. На величину и направление гидродинамического потока влияют направление градиента температуры и характер сцепления ЖК-молекул с ограничивающими поверхностями. Если локальный градиент температуры в объеме ЖК-фазы легко формируется с помощью сфокусированного лазерного излучения, то градиент поля директора удается сформировать посредством гибридной ориентации ЖК-фазы на ограничивающих поверхностях. Все это указывает на то, что существует еще одна возможность немеханической транспортировки микролитровых объемов ЖК-фазы в микроразмерных каналах и капиллярах [6]. Но нужно принять во внимание тот факт, что в микроразмерных объемах ЖК-фазы всегда существует какое-то количество ориентационных дефектов (ОД), которые влияют на процесс транспортировки ЖК-материалов в каналах. Влияние ОД на гидродинамические течения в микроразмерных ЖК-каналах, формирующиеся в результате взаимодействия градиентов температуры и поля директора, будет исследовано в рамках нелинейного обобщения классической теории Эриксена-Лесли [7,8], с учетом баланса энтропии [9]. Численными методами будут исследованы различные гидродинамические режимы, возникающие в ЖК-ячейках под действием сфокусированного лазерного излучения. 


\section{2. Основные гидродинамические уравнения}

Рассмотрим квадратную $2 d \times 2 d \quad$ ЖК-ячейку, ограниченную твердыми горизонтальными и вертикальными поверхностями. Допустим, что директор $\hat{\mathbf{n}}=$ $=n_{x} \hat{\mathbf{i}}+n_{z} \hat{\mathbf{k}}$ планарно сориентирован на верхней и двух вертикальных поверхностях, в то время как на нижней ограничивающей поверхности существует ориентационный дефект. В нашем случае система координат отсчитывается от середины ЖК-ячейки так, что ось $X$ и орт $\hat{\mathbf{i}}$ совпадают с направлением директора на верхней горизонтальной поверхности $\left(\hat{\mathbf{i}} \| \hat{\mathbf{n}}_{\mathrm{z}=\mathrm{d}}\right)$, в то время как ось $Z$ и орт $\hat{\mathbf{k}}$ направлены ортогонально $\left(\hat{\mathbf{k}} \perp \hat{\mathbf{n}}_{\mathrm{z}=\mathrm{d}}\right)$, а орт $\hat{\mathbf{j}}=\hat{\mathbf{k}} \times \hat{\mathbf{i}}$. Таким образом, вектор поля директора $\hat{\mathbf{n}}=\left(n_{x}, 0, n_{z}\right)=n_{x} \hat{\mathbf{i}}+n_{z} \hat{\mathbf{k}}=\cos \theta(x, z, t) \hat{\mathbf{i}}+\sin \theta(x, z, t) \hat{\mathbf{k}}$ лежит в плоскости $x O z$, причем $\theta$-полярный угол образованный директором $\hat{\mathbf{n}}$ и ортом $\hat{\mathbf{k}}$. Дальнейшее изложение будет дано для случая безразмерных переменных $\bar{x}=x / d$ и $\bar{z}=z / d$, причем верхняя черта над пространственными переменными в дальнейшем будет опущена. Поскольку основной нашей целью является исследование влияния ОД на гидродинамический поток, возникающий в результате взаимодействия градиентов температуры $\nabla T$ и поля директора $\nabla \hat{\mathbf{n}}$, то рассмотрим ОД, допускающий существование $\nabla \theta$ вдоль нижней ограничивающей поверхности, то есть,

$$
\begin{gathered}
\theta_{-1 \leq x \leq-L, z=-1}=\theta_{L \leq x \leq 1, z=-1}=0, \\
\theta_{-L<x<L, z=-1}=\tan ^{-1}\left(\frac{L^{2}-x^{2}}{4 x^{4}}\right)(\text { Case I), }
\end{gathered}
$$

и случай „бездефектной“ нижней гомеотропно ориентированной ограничивающей поверхности

$$
\theta_{-1 \leq x \leq-1, z=-1}=0 \text { (Case II) }
$$

соответственно. Здесь $L-$ линейный размер ОД на нижней ограничивающей поверхности. На всех остальных поверхностях граничные условия для полярного угла $\theta$ могут быть записаны в виде

$$
\begin{gathered}
\theta_{x=1,-1<z<1}=\theta_{x=-1,-1<z<1}=0, \\
\theta_{-1 \leq x \leq 1, z=1}=\frac{\pi}{2} .
\end{gathered}
$$

Далее предположим, что через нижнюю ограничивающую поверхность осуществляется поток тепла $\mathbf{q}=q_{z} \hat{\mathbf{k}}$, который в безразмерной форме может быть записан в виде

$$
\left(\frac{\partial \chi(x, z, \tau)}{\partial z}\right)_{z=-1}=\left[\frac{q_{z}-(\lambda-1) \sin 2 \theta \chi, x}{\lambda\left(\cos ^{2} \theta+\sin ^{2} \theta\right)}\right]_{z=-1}
$$

в то время как на всех остальных поверхностях температура поддерживается постоянной, то есть,

$$
\chi_{-1 \leq x \leq 1, z=1}=\chi_{x=-1,-1<z<1}=\chi_{x=1,-1<z<1}=\chi_{1} .
$$

Здесь $q_{z}=Q(x, \tau)$ - поток тепла через нижнюю границу, $\lambda=\lambda_{\|} / \lambda_{\perp}-$ отношение коэффициентов теплопроводности вдоль и поперек направления директора, $\quad \chi \equiv \chi(x, z, \tau)=T(x, z, \tau) / T_{\mathrm{NI}}-$ безразмерная температура, $T_{\mathrm{NI}}-$ температура перехода нематик-изотропной жидкости, $\tau=\left(\frac{K_{1}}{\gamma_{1} d^{2}}\right) t-$ безразмерное время, $K_{1}$ и $\gamma_{1}-$ коэффициенты упругости продольного изгиба и вращательной вязкости соответственно, $Q=-\frac{\bar{q} d}{T_{\mathrm{NI}} \lambda \downarrow}$ и $\bar{q}-$ безразмерная и размерная величины потока тепла через нижнюю ограничивающую поверхность соответственно.

Следует отметить, что в случае отсутствия ОД на нижней ограничивающей поверхности, когда $\theta_{z=-1}=0$, уравнение (4) принимает вид

$$
\left(\frac{\partial \chi(x, z, \tau)}{\partial z}\right)_{z=-1}=q_{z}
$$

Поле скорости $\mathbf{v}=u \hat{\mathbf{i}}+w \hat{\mathbf{k}}$ подчиняется условию прилипания на твердых ограничивающих поверхностях

$$
\begin{gathered}
u_{-1 \leq x \leq 1, z=-1}=u_{x=-1,-1<z<1}=u_{x=1,-1<z<1} \\
=u_{-1 \leq x \leq 1, z=1}=0, \\
w_{-1 \leq x \leq 1, z=-1}=w_{x=-1,-1<z<1}=w_{x=1,-1<z<1} \\
=w_{-1 \leq x \leq 1, z=1}=0,
\end{gathered}
$$

где $u \equiv v_{x}(x, z, \tau)$ и $w \equiv v_{z}(x, z, \tau)$ являются компонентами вектора скорости v. Принимая во внимание микроскопические размеры ЖК-ячейки, мы можем предположить, что плотность ЖК-системы $\rho$ постоянна и мы имеем дело с несжимаемой жидкостью. Это позволяет нам записать уравнение сохранения $\nabla \cdot \mathbf{v}=0$ в виде

$$
u_{, x}+w_{, z}=0,
$$

где $u_{, x}=\frac{\partial u}{\partial x_{1}}$.

Баланс моментов и импульсов, действующий на единицу объема ЖК-фазы, может быть записан в виде [6]:

$$
\begin{gathered}
\theta_{, \tau}=\frac{1}{2}\left(u_{, z}-w_{, x}\right)+\frac{\gamma_{21}}{2} \sin 2 \theta\left(u_{, x}-w_{, z}\right)+\gamma_{21} \cos 2 \theta \\
\times\left(u_{, z}+w_{, x}\right)-u \theta_{, x}-w \theta_{, z}+\left(\cos ^{2} \theta+K_{31} \sin ^{2} \theta\right) \theta_{, x x} \\
+\left(\sin ^{2} \theta+K_{31} \cos ^{2} \theta\right) \theta_{, z z}+\left(K_{31}-1\right) \sin 2 \theta \\
\times\left(\theta_{, x z}+\frac{1}{2}\left(\theta_{, x}^{2}-\theta_{, z}^{2}\right)\right)+\left(K_{31}-1\right) \cos 2 \theta \theta_{, x} \theta_{, z} \\
+\delta_{1} \chi_{, x}\left[-\frac{1}{4} \sin 2 \theta \theta_{, z}+\left(1+\frac{1}{2} \sin ^{2} \theta\right) \theta_{, x}\right] \\
+\delta_{1} \chi_{, z}\left[-\frac{1}{4} \sin 2 \theta \theta_{, x}+\left(1+\frac{1}{2} \cos ^{2} \theta\right) \theta_{, z}\right] \\
\delta_{2} \frac{d u}{d \tau}=\sigma_{x x, x}+\sigma_{z x, z}, \\
\delta_{2} \frac{d w}{d \tau}=\sigma_{z z, z}+\sigma_{x z, x}
\end{gathered}
$$

соответственно. Здесь $K_{31}=K_{3} / K_{1}$ - отношение коэффициентов упругости поперечного и продольного из- 
гибов, $\gamma_{21}=\gamma_{2} / \gamma_{1}-$ отношение коэффициентов вращательной вязкости, $\frac{d u}{d \tau}-$ материальная производная компоненты скорости и, $\sigma_{i j}=P \delta_{i j}+\sigma_{i j}^{\mathrm{el}}+\sigma_{i j}^{\mathrm{vis}}+\delta_{1} \sigma_{i j}^{\mathrm{tm}}$ $(i, j=x, z)$ - безразмерный $\mathrm{TH}$, состоящий из упругого $\sigma_{i j}^{\mathrm{el}}$, вязкого $\sigma_{i j}^{\mathrm{vis}}$ и термомеханического $\sigma_{i j}^{\mathrm{tm}}$ вкладов соответственно, $P$ - гидростатическое давление в ЖК-системе, а $\delta_{1}=\xi \frac{T_{\mathrm{NI}}}{K_{1}}$ и $\delta_{2}=\frac{\rho K_{1}}{\gamma_{1}^{2}}-$ два параметра ЖК-системы.

Безразмерное уравнение баланса энтропии может быть записано в виде [6,9]:

$$
\begin{aligned}
\delta_{3} \frac{\partial \chi}{\partial \tau}= & \mathscr{D}_{1} \chi_{, x x}+\mathscr{D}_{2} \chi_{, z z}+2 \mathscr{D}_{3} \chi_{, x z}+\mathscr{D}_{1, x} \chi_{, x}+\mathscr{D}_{2, z} \chi_{, z} \\
& -\mathscr{D}_{3, x} \chi_{, z}-\mathscr{D}_{3, z} \chi_{, x}-\delta_{3}\left(u \chi_{, x}+w \chi_{, z}\right),
\end{aligned}
$$

где $\mathscr{D}_{1}=\lambda \sin ^{2} \theta^{2}+\cos ^{2} \theta, \mathscr{D}_{2}=\lambda \cos ^{2} \theta+\sin ^{2} \theta$ и $\mathscr{D}_{3}=$ $=\frac{\lambda-1}{2} \sin 2 \theta-$ функции пространственных переменных $x$ и $z$ и времени $\tau, \chi_{, x}=\frac{\partial \chi}{\partial x}, \chi_{, x x}=\frac{\partial^{2} \chi}{\partial x^{2}}, \delta_{3}=\frac{\rho C_{p} K_{1}}{\gamma_{1} \lambda_{\perp}}-$ еще один параметр системы, и $\xi \sim 10^{-12} \mathrm{~J} / \mathrm{mK}$ - термомеханическая постоянная [5], а $C_{p}-$ теплоемкость ЖК-системы.

Будем предполагать, что поток тепла осуществляется за счет лазерного излучения, сфокусированного на нижней горизонтальной поверхности, и может быть записан в виде

$$
Q=\bar{Q} \exp \left(-\frac{2\left(x-x_{0}\right)^{2}}{\bar{\omega}_{0}^{2}}\right) \mathscr{H}\left[\tau_{\text {in }}-\tau\right]
$$

где $Q-$ безразмерный поток тепла через нижнюю горизонтальную поверхность, зависящий от энергетических параметров лазерного излучения, $\bar{\omega}_{0}=\frac{\omega_{0}}{d}, \omega_{0}-$ размер гауссового пятна лазерного излучения, $\mathscr{H}\left[\tau_{\text {in }}-\tau\right]-$ функция Хэвисайда, и $\tau_{\text {in }}$ - время инжекции лазерного излучения.

Отметим, что ограничения, налагаемые на энергетические параметры лазерного излучения, вытекают из небольшого температурного интервала существования нематической фазы, образованной молекулами 4-n-пентил- $4^{\prime}$-цианобифенила (5ЦБ). При температуре $300 \mathrm{~K}$ и плотности $\rho=10^{3} \mathrm{~kg} / \mathrm{m}^{3}$ все вышеперечисленные параметры системы уравнений (9)-(11) имеют следующие значения: $\delta_{1} \sim 24, \delta_{2} \sim 2 \cdot 10^{-6}$, и $\delta_{3} \sim 1 \cdot 10^{-3}$ соответственно. Принимая во внимание тот факт, что $\delta_{2} \ll 1$, уравнение (10) может быть переписано в виде [10]:

$$
\begin{aligned}
& a_{1} \psi_{, z z z z}+a_{2} \psi_{, x z z z}+a_{3} \psi_{, x x z z}+a_{4} \psi_{, x x x z} \\
& \quad+a_{5} \psi_{, x x x x}+a_{6} \psi_{, z z z}+a_{7} \psi_{, x z z}+a_{8} \psi_{, x x z} \\
& \quad+a_{9} \psi_{, x x x}+a_{10} \psi_{, z z}+a_{11} \psi_{, x z}+a_{12} \psi_{, z z}+\mathscr{F}=0
\end{aligned}
$$

где $\psi=\frac{\rho C_{p}}{\lambda_{\perp}} \bar{\psi}-$ безразмерный аналог функции тока $\bar{\psi}$ для поля скорости $\mathbf{v}=u \hat{\mathbf{i}}+w \hat{\mathbf{k}}=-\nabla \times \hat{\mathbf{j}} \psi$, а выражение для функции $\mathscr{F}$ и значения коэффициентов $a_{i}(i=1, \ldots, 12)$ приведены в Приложении.
Таким образом, формирование безразмерного поля скорости $\mathbf{v}$, под действием градиента температуры $\nabla \chi$, в микрометровом ЖК-объеме может быть описано системой нелинейных дифференциальных уравнений в частных производных (9), (11) и (13) и дополненной граничными (1)-(7) и начальными условиями как для поля директора и скорости, так и для температуры. Начальные условия для поля скорости и температуры могут быть записаны в виде

$$
\psi(\tau=0, x, z)=0, \quad \chi(\tau=0, x, z)=0.97,
$$

в то время как начальное условие для поля директора имеет вид

$$
\hat{\mathbf{n}}(\tau=0, x, z)=\hat{\mathbf{n}}_{\mathrm{el}}(x, z),
$$

где $\hat{\mathbf{n}}_{\mathrm{el}}=\cos \theta_{\mathrm{el}}(x, z) \hat{\mathbf{i}}+\sin \theta_{\mathrm{el}}(x, z) \hat{\mathbf{k}}$, а значение угла $\theta_{\mathrm{el}}(x, z)$ рассчитано с помощью уравнения (9) при условии, что $u_{, x}=u_{, z}=u_{, x}=w_{, x}=w_{, z}=\chi_{, x}=\chi_{, z}=0$, при том, что начальное условие для угла $\theta_{\mathrm{el}}$ может быть записано в виде

$$
\theta_{\mathrm{el}}(x, z, \tau=0)=\left\{\begin{array}{l}
\frac{\pi}{2} z^{2}, \quad x \in[-L, L] \text { и } z \in[-1,1], \\
0, \quad x \in[-1,-L) \bigcup(L, 1] \text { и } z \in[-1,1] .
\end{array}\right.
$$

\section{3. Решение гидродинамических уравнений и основные результаты}

Формирование гидродинамического потока $\mathbf{v}(\mathbf{r}, t)$ и последовавшая за этим переориентация поля директора $\hat{\mathbf{n}}(\mathbf{r}, t)$ в изначально однородно прогретой ЖК-ячейке под действием градиента температуры $\nabla \chi$, сформированного с помощью лазерного излучения мощностью $\sim 0.5 \mathrm{~W}$ и продолжительностью $\sim 0.18 \mathrm{~ms}$, сфокусированного на нижней ограничивающей поверхности, было исследовано методом релаксации [11] и сеточным методом [12], а критерием сходимости итерационной процедуры был выбран $\epsilon=\left|\left(\chi_{(m+1)}(x, z, \tau)-\chi_{(m)}(x, z, \tau)\right) / \chi_{(m)}(x, z, \tau)\right|$ $\sim 10^{-4}$, где $m$ - число итераций. В начальный момент времени $(\tau=0)$, с помощью уравнения (9) и условия отсутствия течения $u=w=0 \chi_{, x}=\chi_{, z}=0$, а также с учетом начального условия (14) было рассчитано распределение угла $\theta_{\mathrm{el}}(x, z)$, что позволило рассчитать функцию $\mathscr{F}$, распределение поля температуры $\chi(x, z, \Delta \tau)$ и функцию тока $\psi(x, z, \Delta \tau)$, соответствующие следующему моменту времени $\Delta \tau$. Следующий шаг по времени $\Delta \tau$ для распределения поля скорости, температуры и поля директора по всему объему ЖК-ячейки был осуществлен с помощью сеточного метода [12], причем устойчивость численной процедуры определялась из условия

$$
\min _{-1 \leq x \leq 1,-1 \leq z \leq 1}\left[\frac{a_{5}}{a_{1}}\left(\frac{\Delta z}{\Delta x}\right)^{4}\right] \geq \frac{2}{3}
$$

и

$$
\frac{2}{\Delta \tau} \geq \frac{\mathscr{D}_{1}}{(\Delta x)^{2}}+\frac{\mathscr{D}_{2}}{(\Delta z)^{2}}
$$

соответственно. Здесь $\Delta x$ и $\Delta z-$ приращения пространственных переменных, а коэффициенты $a_{1}$ и $a_{5}$ приве- 
дены в Приложении. На рис. 1 представлен результат расчета распределения поля директора $\hat{\mathbf{n}}\left(x, z, \tau=\tau_{\text {in }}\right)$ по обьему ЖК-ячейки для случая, когда был учтен ОД на нижней ограничивающей поверхности (case I), через которую был направлен тепловой поток $q_{z}$, возникающий в результате сфокусированного лазерного излучения мощностью $\sim 0.5 \mathrm{~W}$ и продолжительностью $\tau=10^{-4}(\sim 0.18 \mathrm{~ms})$. Зависимость полярного угла $\theta\left(x, z=-0.98, \tau=\tau_{i n}\right)$ от пространственной переменной $x \in[-1,1]$ вблизи нижней ограничивающей поверхности $(z=-0.98)$, на которую было сфокусировано лазерное излучение в течение времени $\tau=\tau_{\text {in }}$ (рис. $2, a$ ), и распределение угла $\theta\left(x=0, z, \tau=\tau_{i n}\right)$ по сечению $z \in[-1,1]$ ЖК-ячейки (рис. 2,b), свидетельствуют о

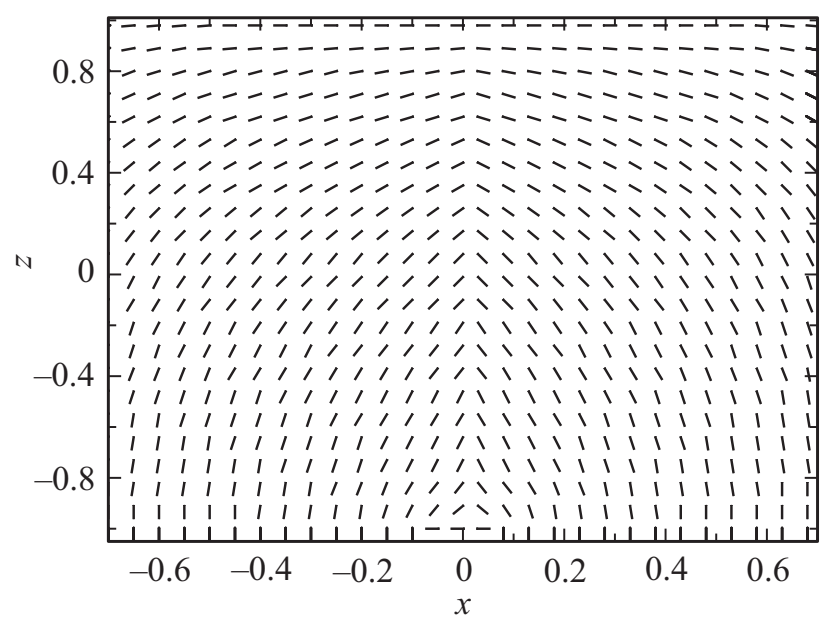

Рис. 1. Распределение поля директора $\hat{\mathbf{n}}\left(x, z, \tau=\tau_{\text {in }}\right)$ по объему ЖК-ячейки под действием сфокусированного лазерного излучения мощностью $\sim 0.5 \mathrm{~W}$ и продолжительностью $\sim 0.18 \mathrm{~ms}$.
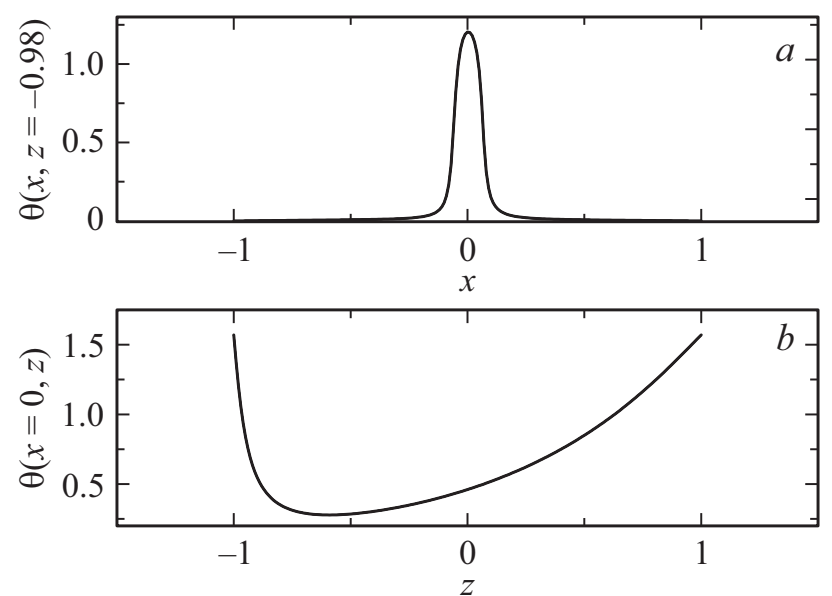

Рис. 2. (a) Равновесное распределение угла $\theta(x, z=-0.98$, $\tau=\tau_{i n}$ ) по ширине ЖК-ячейки $-1 \leq x \leq 1$ вблизи нижней ограничивающей поверхности $z=-0.98$, спустя время $\tau_{5}=\tau_{i n}=10^{-4}(\sim 0.18 \mathrm{~ms})$. (b) То же, что на рис. $2, a$, но распределение угла $\theta\left(x=0, z, \tau=\tau_{i n}\right)$ вдоль оси $-1 \leq z \leq 1$, посередине $(x=0.0)$ ЖК-ячейки.

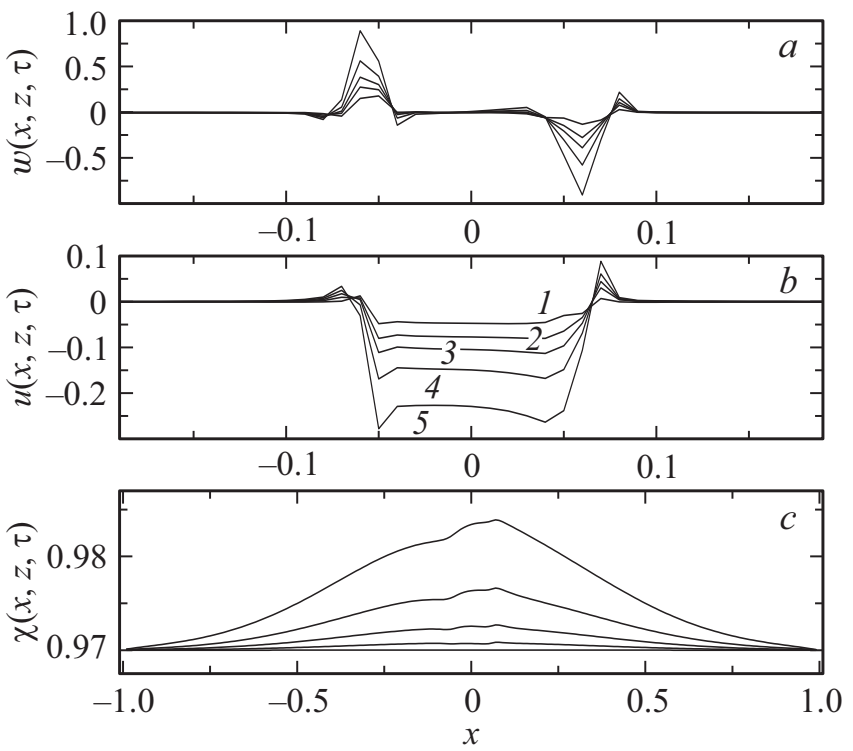

Рис. 3. (а) Эволюция распределения вертикальной составляющей вектора скорости $w(x, z=-0.98, \tau)$ по ширине ЖК-ячейки $-1 \leq x \leq 1$ в процессе ее разогрева лазерным излучением. Результаты даны для 5 значений времени с момента включения лазерного излучения: $\tau_{1}=10^{-7}(\sim 1 \mathrm{~ns})$ (кривая 1$), \tau_{2}=1.6 \cdot 10^{-6}(\sim 2.8 \mu \mathrm{s})$ (кривая 2$), \tau_{3}=6.4 \cdot 10^{-6}$ $(\sim 11.2 \mu \mathrm{s})$ (кривая 3$), \tau_{4}=2.56 \cdot 10^{-5}(\sim 45 \mu \mathrm{s})$ (кривая 4$)$ и $\tau_{5}=\tau_{i n}=10^{-4}(\sim 0.18 \mathrm{~ms})$ (кривая 5) соответственно. (b) То же, что на рис. $3, a$, но для распределения горизонтальной составляющей вектора скорости $u(x, z=-0.98, \tau)$. (c) То же, что на рис. $3, a$, но для распределения температуры $\chi(x, z=-0.98, \tau)$ по ширине ЖК-ячейки.

том, что под действием лазерного излучения основная переориентация поля директора $\hat{\mathbf{n}}(x, z, \tau)$ происходит вблизи ориентационного дефекта. Расчеты, представленные на рис. 1, также свидетельствуют о том, что влияние ОД, который занимает $\sim 5 \%$ ширины нижней ограничивающей поверхности, распространяется вглубь ЖК-образца почти на $80 \%$ его толщины. Процесс формирования поля скорости $\mathbf{v}=u \hat{\mathbf{i}}+w \hat{\mathbf{k}}$ в результате взаимодействия градиентов поля директора $\nabla \hat{\mathbf{n}}$ и температуры $\nabla \chi$, инициируемого лазерным излучением, представлен на рис. 3, $a$ и $b$, в то время как перераспределение поля температуры $\chi$ представлено на рис. 3, $c$ соответственно. Эволюция как горизонтальной $u$, так и вертикальной $w$ составляющих вектора скорости $\mathbf{v}$ в процессе разогрева ЖК-фазы свидетельствует о том, что вблизи ОД формируется вихревой поток (см. рис. 4) с центром в точке $x_{0}=0.0$ и $z_{0} \sim-0.94$. Отметим, что на рис. 4 $1 \mathrm{~mm}$ длины стрелки соответствует скорость $1.8 \mu \mathrm{m} / \mathrm{s}$. Этот вихревой поток охватывает небольшую область ЖК-фазы, прилегающую к ОД на нижней ограничивающей поверхности $(-L \leq x \leq L,-1.0 \leq z \leq-0.94)$ (см. рис. 4). При этом максимальные значения вертикальной составляющей вектора скорости достигаются вблизи краев ОД $w(x \sim \pm L, z, \tau)$ и направлены в противоположные стороны так, чтобы формировалось вихревое 


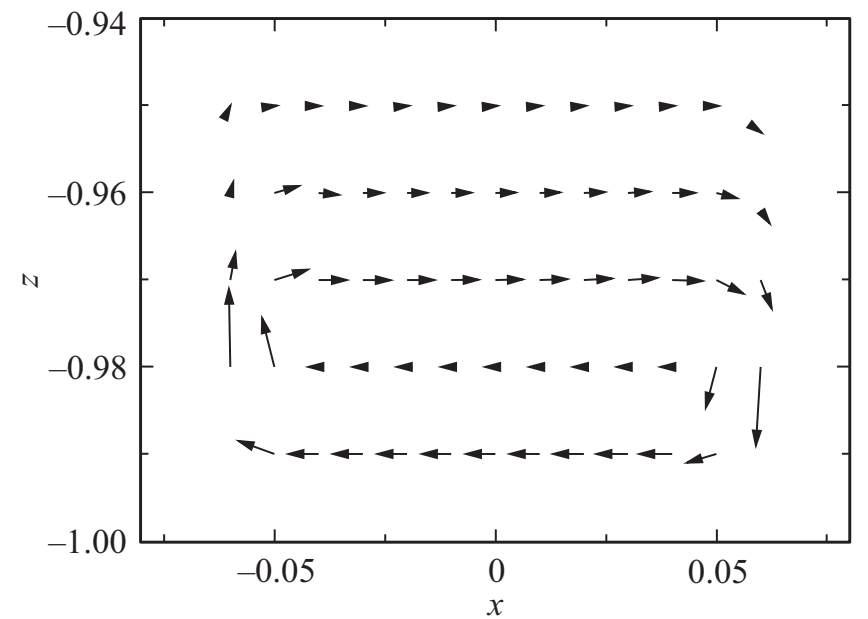

Рис. 4. Распределение поля скорости $\mathbf{v}\left(x, z, \tau=\tau_{\text {in }}\right)$ по объему „дефектной“ ЖК-ячейки спустя время $\tau=\tau_{\text {in }}=$ $=10^{-4}(\sim 0.18 \mathrm{~ms})$ с начала прогревания. Здесь $1 \mathrm{~mm}$ длины стрелки соответствует скорость $\sim 1.8 \mu \mathrm{m} / \mathrm{s}$.

течение, направленное по часовой стрелке (см. рис. 3, $a$ и 4). Следует отметить, что эволюция температуры в процессе разогрева ЖК-фазы описывается слегка асимметричным профилем $\chi(x, z, \tau)$ относительно середины ЖК-ячейки $(x=0.0)$, что обусловлено наличием $\nabla \theta$ на нижней ограничивающей поверхности (см. рис. $3, c$ ).

С момента выключения лазерного излучения начинается процесс охлаждения ЖК-фазы и, как следствие, затухание вихревого потока (см. рис. 5, $a$ и $b$ ). На pис. $5, a, b$ и $c$ показана эволюция компонент вектора скорости $u(x, z=-0.98, \tau)$ и $w(x, z=-0.98, \tau)$, а также температуры $\chi(x, z=-0.98, \tau)$ по ширине ЖК-ячейки $-1 \leq x \leq 1$ для 5 значений времени с момента выключения лазерного излучения: $\tau_{6}=0.5 \cdot 10^{-4}(\sim 0.09 \mathrm{~ms})$ (кривая 6$), \quad \tau_{7}=3.2 \cdot 10^{-4} \quad(\sim 0.58 \mathrm{~ms}) \quad$ (кривая 7), $\tau_{8}=7.8 \cdot 10^{-4}(\sim 1.34 \mathrm{~ms}) \quad($ кривая 8$), \tau_{9}=1.4 \cdot 10^{-3}$ $(\sim 2.7 \mathrm{~ms}) \quad$ (кривая 9$) \quad$ и $\quad \tau_{10}=\tau_{R}=6.2 \cdot 10^{-3}$ ( $\sim 11.6 \mathrm{~ms}$ ) (кривая 10 ) соответственно. Таким образом, гидродинамическое течение, инициированное лазерным разогревом, полностью затухает спустя $\sim 11.6 \mathrm{~ms}$ после выключения лазера, а температурное поле по всему ЖК-объему выравнивается и принимает значение, которое задано на ограничивающих поверхностях. Чтобы понять, как влияет ОД, расположенный на нижней ограничивающей поверхности (case I), на процесс формирования гидродинамического течения в ЖК-ячейке под действием лазерного излучения, рассмотрим случай „бездефектного“ ЖК-образца (case II). Процесс формирования поля скорости $\mathbf{v}=u \hat{\mathbf{i}}+w \hat{\mathbf{k}}$, возникающего в результате взаимодействия градиентов поля директора $\nabla \hat{\mathbf{n}}$ и температуры $\nabla \chi$, в „бездефектном“ объеме ЖК-фазы представлен на рис. 6 . Эволюция как горизонтальной $u$, так и вертикальной $w$ составляющих вектора скорости в процессе разогрева ЖК-образца свидетельствуют о формировании вихревого течения вблизи нижней ограничива- ющей поверхности. Расчеты показывают, что в вихревой поток, создаваемый лазерным излучением мощностью $\sim 0.5 \mathrm{~W}$ и продолжительностью $\tau=10^{-4}(\sim 0.18 \mathrm{~ms})$, вовлечена незначительная область ЖК-фазы, ограниченная размерами $-1.0 \leq z \leq-0.94$ и $-0.4 \leq x \leq 0.4$, или $\sim 5 \%$ всего объема ЖК-фазы. Несмотря на то, что размеры этого вихря сопоставимы с размерами
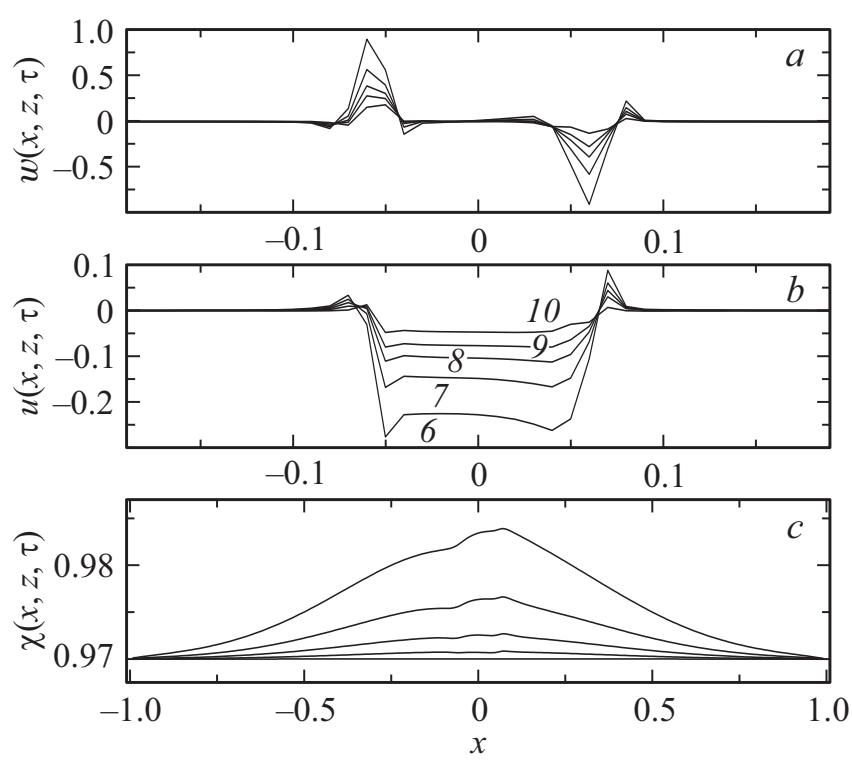

Рис. 5. Релаксация распределения вертикальной $w(x, z=-0.98, \tau) \quad(a)$ и горизонтальной $u(x, z=-0.98, \tau) \quad(b)$ составляющих вектора скорости, а также температуры $\chi(x, z=-0.98, \tau)$ (c) по ширине ЖК-ячейки $-1 \leq x \leq 1$ в процессе ее охлаждения. Результаты даны для 5 значений времени с момента выключения лазерного излучения: $\tau_{6}=0.5 \cdot 10^{-4}(\sim 0.09 \mathrm{~ms})$ (кривая 6), $\tau_{7}=3.2 \cdot 10^{-4} \quad(\sim 0.58 \mathrm{~ms}) \quad($ кривая $\quad 7), \quad \tau_{8}=7.8 \cdot 10^{-4}$ $(\sim 1.34 \mathrm{~ms})$ (кривая 8$), \tau_{9}=1.4 \cdot 10^{-3}(\sim 2.7 \mathrm{~ms})$ (кривая 9) и $\tau_{10}=\tau_{R}=6.2 \cdot 10^{-3}(\sim 11.6 \mathrm{~ms})$ (кривая 10$)$ соответственно.

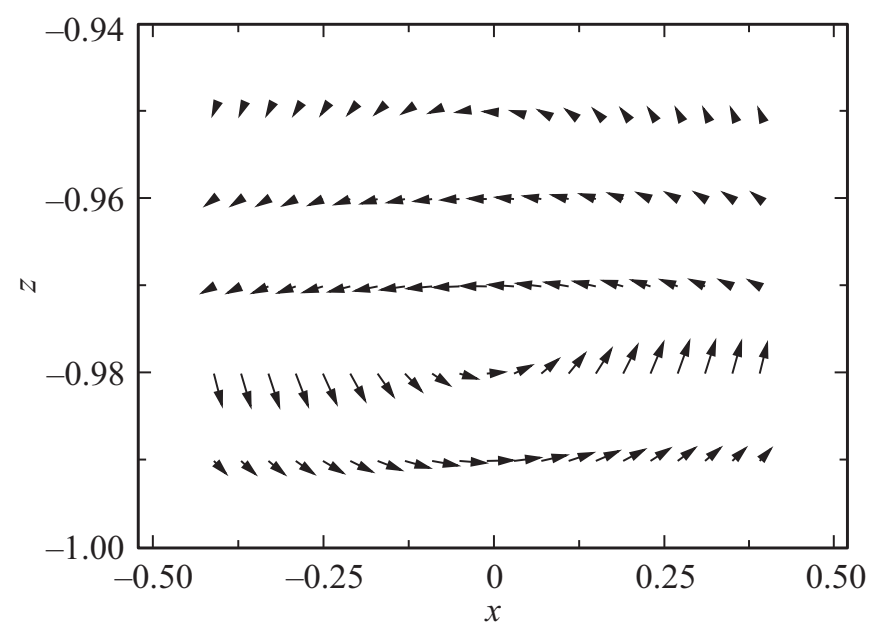

Рис. 6. То же, что на рис. 4, но для „бездефектной“ ЖК-ячейки, спустя время $\tau=\tau_{R} \sim 6.2 \cdot 10^{-3}(\sim 11.6 \mathrm{~ms})$ с момента отключения лазерного излучения. 
вихря, возникающего в „дефектном“ ЖК-объеме, скорость вращения „дефектного“ вихря на 2 порядка превосходит скорость вращения „бездефектного“ вихря. Так, максимальные значения безразмерных скоростей $u$ и $w$ для случая II, спустя время $\tau_{\text {in }}=10^{-4}(\sim 0.18 \mathrm{~ms})$, равны $1.4 \cdot 10^{-3}(\sim 40 \mathrm{~nm} / \mathrm{s})$ и $3.7 \cdot 10^{-5}(\sim 1 \mathrm{~nm} / \mathrm{s})$ соответственно, в то время как для случая I значения этих скоростей равны $0.28(\sim 7.8 \mu \mathrm{m} / \mathrm{s})$ и $0.9(\sim 25.5 \mu \mathrm{m} / \mathrm{s})$ соответственно. Таким образом, расчеты показывают, что наличие ориентационных дефектов в ЖК-ячейке может принципиально изменить характер и величину гидродинамического потока, возникающего в ЖК-фазе под действием градиента температуры. Но учитывая тот факт, что в случае „дефектной“ ЖК-ячейки максимальные величины компонент скорости вихревого потока, возникающего в ЖК-ячейке в результате разогрева ее сфокусированным лазерным излучением, столь незначительны, то это поле скорости практически не влияет на процесс переориентации поля директора в этой ячейке.

\section{4. Заключение}

Анализ полученных результатов показал, что в ЖК-ячейке с ориентационным дефектом (ОД), расположенным на нижней ограничивающей поверхности („дефектной“ ЖК-ячейки), которая была подвергнута локальному разогреву с помощью сфокусированного лазерного излучения, возможно формирование вихревого потока вблизи ОД, причем скорость вращения этого вихря на два порядка превосходит скорость вращения вихря, формирующегося при тех же условиях в „бездефектном“ ЖК-объеме.

Исследованные в работе особенности, связанные с реакцией ЖК-материала, инкапсулированного в микроразмерную ячейку, на локализованное воздействие градиента температуры, необходимо учитывать при создании сенсоров и датчиков, используемых в биотехнологических приложениях, медицине и фармакологии, а также в иммерсионной литографии.

\section{5. Приложение}

Коэффициенты $a_{i}(i=1, \ldots 12)$ имеют вид

$a_{1}=\frac{1}{4 \gamma_{1}}\left[\alpha_{1} \sin ^{2} 2 \theta+2 \alpha_{4}+\alpha_{5}+\alpha_{6}+\gamma_{1}-2 \gamma_{2} \cos 2 \theta\right]$,

$$
a_{2}=-\frac{\gamma_{2}}{\gamma_{1}} \sin 2 \theta
$$

$a_{3}=\frac{1}{4 \gamma_{1}}\left[4 \alpha_{4}+2 \alpha_{5}+2 \alpha_{6}+2 \gamma_{1}+\alpha_{1}(1+3 \cos 4 \theta)\right]$, $a_{4}=a_{2}$,

$a_{5}=\frac{1}{4 \gamma_{1}}\left[\alpha_{1} \sin ^{2} 2 \theta+2 \alpha_{4}+\alpha_{5}+\alpha_{6}+\gamma_{1}+2 \gamma_{2} \cos 2 \theta\right]$,

$$
\begin{aligned}
& a_{6}=\frac{1}{\gamma_{1}}\left[\theta_{, z}\left(\alpha_{1} \sin 4 \theta+\gamma_{2} \sin 2 \theta\right)\right. \\
& \left.+\theta_{, x}\left(\alpha_{1} \cos 4 \theta-\gamma_{2} \cos 2 \theta\right)\right], \\
& a_{7}=-\frac{1}{\gamma_{1}}\left[\theta_{, z}\left(\alpha_{1} \cos 4 \theta+3 \gamma_{2} \cos 2 \theta\right)+\theta_{, x}\left(3 \alpha_{1} \sin 4 \theta\right)\right] \text {, } \\
& a_{8}=\frac{1}{\gamma_{1}}\left[\theta_{, z}\left(-3 \alpha_{1} \sin 4 \theta\right)+\theta_{, x}\left(\alpha_{1} \cos 4 \theta-3 \gamma_{2} \cos 2 \theta\right)\right] \text {, } \\
& a_{9}=-\frac{1}{\gamma_{1}}\left[\theta_{, z}\left(2 \alpha_{1} \cos 4 \theta+2 \gamma_{2} \cos 2 \theta\right)\right. \\
& \left.+\theta_{, x}\left(\alpha_{1} \sin 4 \theta-2 \gamma_{2} \sin 2 \theta\right)\right], \\
& a_{10} \gamma_{1}=\theta_{, x x}\left(-\frac{\alpha_{1}}{2} \sin 4 \theta\right)+\theta_{, x z}\left(\alpha_{1} \cos 4 \theta-\gamma_{2} \cos 2 \theta\right) \\
& -2 \theta_{, x}^{2}\left(\alpha_{1} \cos 4 \theta\right)+\theta_{, z z}\left(\frac{\alpha_{1}}{2} \sin 4 \theta+\gamma_{2} \sin 2 \theta\right) \\
& -\theta_{, x} \theta_{, z}\left(4 \alpha_{1} \sin 4 \theta+2 \gamma_{2} \sin 2 \theta\right) \\
& +2 \theta_{, z}^{2}\left(\alpha_{1} \cos 4 \theta+\gamma_{2} \cos 2 \theta\right) \\
& a_{11} \gamma_{1}=\theta_{, x x}\left(\alpha_{1} \cos 4 \theta-\gamma_{2} \cos 2 \theta\right)-2 \theta_{, x z}\left(\alpha_{1} \sin 4 \theta\right) \\
& +\theta_{, x}^{2}\left(2 \alpha_{1} \sin 4 \theta+\gamma_{2} \sin 2 \theta\right)-\theta_{, z z}\left(\alpha_{1} \cos 4 \theta+\gamma_{2} \cos 2 \theta\right) \\
& -\theta_{, x} \theta_{, z}\left(8 \alpha_{1} \sin 4 \theta\right)+2 \theta_{, z}^{2}\left(2 \alpha_{1} \sin 4 \theta+\gamma_{2} \sin 2 \theta\right), \\
& a_{12} \gamma_{1}=\theta_{, x x}\left(\frac{\alpha_{1}}{2} \sin 4 \theta-\gamma_{2} \sin 2 \theta\right) \\
& -\theta_{, x z}\left(\alpha_{1} \cos 4 \theta+\gamma_{2} \cos 2 \theta\right)+2 \theta_{, x}^{2}\left(\alpha_{1} \cos 4 \theta+\gamma_{2} \cos 2 \theta\right) \\
& -\theta_{, z z}\left(\frac{\alpha_{1}}{2} \sin 4 \theta\right)+2 \theta_{, x} \theta_{, z}\left(2 \alpha_{1} \sin 4 \theta+\gamma_{2} \sin 2 \theta\right) \\
& -2 \theta_{, z}^{2}\left(\alpha_{1} \cos 4 \theta\right) \text {. }
\end{aligned}
$$

Компоненты функции $\mathscr{F}=\mathscr{F}_{1, z}-\mathscr{F}_{2, x}$ имеют вид

$$
\mathscr{F}_{1}=\left(f^{1, t m} \chi_{, x}\right)_{, x}+\left(f^{3, t m} \chi_{, x}\right)_{, z}+\left(f^{4, t m} \chi_{, z}\right)_{, z}
$$

и

$$
\mathscr{F}_{2}=-\left(f^{2, t m} \chi_{, z}\right)_{, z}+\left(f^{5, t m} \chi_{, x}\right)_{, x}+\left(f^{6, t m} \chi_{, z}\right)_{, x} .
$$

Здесь

$$
\begin{aligned}
f^{1, t m}= & \theta_{, x}\left(\sin 2 \theta+\frac{1}{16} \sin 4 \theta\right) \\
& +\theta_{, z}\left(-\frac{7}{16}+\frac{1}{8} \cos 2 \theta+\frac{1}{16} \cos 4 \theta\right), \\
f^{2, t m}= & \theta_{, x}\left(-\frac{7}{16}-\frac{1}{8} \cos 2 \theta+\frac{1}{16} \cos 4 \theta\right) \\
& +\theta_{, z}\left(\sin 2 \theta-\frac{1}{16} \sin 4 \theta\right),
\end{aligned}
$$




$$
\begin{aligned}
f^{3, t m}= & \theta_{, x}\left(-\frac{13}{32}+\frac{3}{8} \cos 2 \theta+\frac{1}{32} \cos 4 \theta\right) \\
& +\theta_{, z}\left(\frac{1}{16} \sin 2 \theta-\frac{1}{32} \sin 4 \theta\right), \\
f^{4, t m}= & \theta_{, x}\left(\frac{3}{16} \sin 2 \theta-\frac{1}{32} \sin 4 \theta\right) \\
+ & \theta_{, z}\left(-\frac{27}{32}+\frac{5}{8} \cos 2 \theta-\frac{1}{32} \cos 4 \theta\right), \\
f^{5, t m}= & \theta_{, x}\left(-\frac{27}{32}+\frac{5}{8} \cos 2 \theta+\frac{1}{32} \cos 4 \theta\right) \\
& +\theta_{, z}\left(-\frac{3}{16} \sin 2 \theta-\frac{1}{32} \sin 4 \theta\right), \\
f^{6, t m}= & \theta_{, x}\left(-\frac{1}{16} \sin 2 \theta-\frac{1}{32} \sin 4 \theta\right) \\
& +\theta_{, z}\left(\frac{13}{32}+\frac{3}{8} \cos 2 \theta-\frac{1}{32} \cos 4 \theta\right),
\end{aligned}
$$

а $\alpha_{i}(i=1, \ldots, 6)$ - коэффициенты вязкости Лесли.

\section{Список литературы}

[1] S. Lee, R. An, J.A. Hunt. Nature Nanotech. 5, 412 (2010).

[2] E. Verneuil, M.L. Cordero, F. Gallaire, Ch.N. Baroud. Langmuir 25, 5127 (2009).

[3] P.G. de Gennes, J. Prost. The Physics of Liquid Crystals. Oxford Univ. Press., Oxford (1995). 400 p.

[4] Р.С. Акопян, Б.Я. Зельдович. ЖЭТФ 87, 1660 (1984).

[5] R.S. Akopyan, R.B. Alaverdian, E.A. Santrosian, Y.S. Chilingarian. J. Appl. Phys. 90, 3371 (2001).

[6] A. V. Zakharov, A. A. Vakulenko. J. Chem. Phys. 127, 084907 (2007).

[7] J.L. Ericksen. Arch. Ration. Mech. Anal. 4, 231 (1960).

[8] F.M. Leslie. Arch. Ration. Mech. Anal. 28, 265 (1968).

[9] С. Гроот, П. Мазур. Неравновесная термодинамика. Мир, M. (1964). $456 \mathrm{c}$.

[10] А.В. Захаров, А.А. Вакуленко. ФТТ 54, 797 (2012).

[11] И.С. Березин, Н.Р. Жидков. Методы вычислений. Физматгиз, М. (1964). 464 с.

[12] А.А. Самарский, Е.С. Николаев. Методы решения сеточных уравнений. Наука, М. (1978). 592 с.

Редактор Т.Н. Василевская 